\title{
Unidade de terapia intensiva adulto: Percepção da equipe de enfermagem sobre o cuidado ao paciente grave
}

\author{
Adult intensive therapy unit: Nursing staff's perception of Serious Patient Care \\ Unidad de terapia intensiva adulto: Percepción del equipo de enfermería sobre el cuidado \\ del paciente grave \\ Josiane Morais Bezerra ${ }^{1 *}$, Ivana Annely Cortez Fonseca ${ }^{1}$.
}

\begin{abstract}
RESUMO
Objetivo: Descrever a percepção da equipe de enfermagem quanto ao cuidado ao paciente grave em uma UTI adulto em um hospital privado de Porto Velho-RO. Métodos: Trata-se de um estudo de caráter exploratório-descritiva, com métodos de pesquisa bibliográfica e de campo, com enfoque prospectivo, sustentada por abordagem qualitativa. Como instrumento para coleta de dados, utilizou-se um questionário contendo perguntas abertas. Para análise dos resultados, procedeu-se a análise de conteúdo. Os dados obtidos foram analisados por método que consiste em leituras repetidas das respostas dos entrevistados, sua separação em categorias e, por fim, sua análise. O estudo foi aprovado por Comitê de Ética em Pesquisa. Resultados: Evidenciou-se que o estudo contribuiu para reflexões acerca da importância sobre as ações da equipe de enfermagem, com cuidado com os pacientes graves, podendo no futuro, contribuir com as discussões sobre assistência desses profissionais da saúde e a necessidade de um investimento no aprimoramento continuado, preparo, e formação especializada. Conclusão: Conclui-se que os profissionais de enfermagem defendem a humanização como respeito pelo ser humano, assistir ao paciente grave de forma holística e valorizar o paciente e sua família.
\end{abstract}

Palavras-Chave: Percepção, Cuidados, Enfermagem, UTI.

\begin{abstract}
Objective: To describe the perception of the nursing team regarding the care of the critically ill patient in an adult ICU in a private hospital in Porto Velho-RO. Methods: This is an exploratory-descriptive study, with methods of bibliographical and field research, with a prospective approach, supported by a qualitative approach. As a tool for data collection, a questionnaire containing open questions was used. To analyze the results, the content analysis was performed. The data obtained were analyzed by a method consisting of repeated readings of respondents' responses, their separation into categories and, finally, their analysis. The study was approved by the Research Ethics Committee. Results: It was evidenced that the study contributed to reflections about the importance of the actions of the nursing team, with care with the serious patients, being able to contribute in the future to the discussions about the assistance of these health professionals and the need for an investment in the continuous improvement, preparation, and specialized training. Conclusion: It is concluded that nursing professionals defend humanization as a respect for the human being, assist the patient in a holistic way and value the patient and his family.
\end{abstract}

Keywords: Perception, Care, Nursing, UTI.

${ }^{1}$ Faculdade Interamericana de Rondônia (UNIRON), Porto Velho - RO.

*E-mail: josiane_16_pvh@hotmail.com

SUBMETIDO EM: 6/2019

ACEITO EM: 7/2019

PUBLICADO EM: $\mathbf{8 / 2 0 1 9}$

REAS/EJCH | Vol.Sup.31 | e1060 | DOI: https://doi.org/10.25248/reas.e1060.2019 Página 1 de 11 


\section{RESUMEN}

Objetivo: Describir la percepción del equipo de enfermería en cuanto al cuidado al paciente grave en una UTI adulto en un hospital privado de Porto Velho-RO. Métodos: Se trata de un estudio de carácter exploratorio-descriptivo, con métodos de investigación bibliográfica y de campo, con enfoque prospectivo, sustentada por abordaje cualitativo. Como instrumento para la recolección de datos, se utilizó un cuestionario que contenía preguntas abiertas. Para el análisis de los resultados, se procedió a análisis de contenido. Los datos obtenidos fueron analizados por método que consiste en lecturas repetidas de las respuestas de los entrevistados, su separación en categorías y, finalmente, su análisis. El estudio fue aprobado por el Comité de Ética en Investigación. Resultados: Se evidenció que el estudio contribuyó a reflexiones acerca de la importancia sobre las acciones del equipo de enfermería, con cuidado con los pacientes graves, pudiendo en el futuro, contribuir con las discusiones sobre asistencia de estos profesionales de la salud y la necesidad de una inversión en el desarrollo mejora continua, preparación, y formación especializada. Conclusión: Se concluye que los profesionales de enfermería defienden la humanización como respeto por el ser humano, asistir al paciente grave de forma holística y valorar al paciente y su familia.

Palabras clave: Percepción, Cuidados, Enfermería, UTI.

\section{INTRODUÇÃO}

As Unidades de Terapia Intensiva (UTIs) são reservadas aos cuidados especializados aos pacientes em estado críticos e, para os quais, é preciso realizar um controle severo dos padrões vitais, assistência intensivas, entendimento prático e técnico e assistência de enfermagem continuada (LINO MM; SILVA GF, 2013).

O cliente em estado grave perde a convivência direta com seus familiares, é afastado por um tempo indeterminado da sociedade, de suas obrigações e costumes, relacionando-se com pessoas desconhecidas e exposto a acontecimentos inoportunos, além de se encontrar com outros pacientes, em circunstâncias péssimas do que a sua. $O$ paciente pode encarar ainda, causas que geram medo e angústia deixando o paciente debilitado e abatido no seu estado emocional (NASCIMENTO ERP e TRENTINI MO, 2012).

Acredita-se que o cuidado precisa ser sistematizado e holístico, para promover a qualidade da assistência. O processo de cuidar não pode ser pautado apenas na identificação dos sinais e sintomas clínicos da doença, mas nas modificações que ocorrem na estrutura dos seres humanos as quais o abalam em sua totalidade (LINO MM e SILVA SC, 2013).

A UTI é um âmbito da saúde que admite pacientes em situações críticas que tem a necessidade de intensa monitorização, com finalidade de reverter seu estado clínico, melhorando as possibilidades de reabilitação e sobrevivência. Este é um setor que carece da dedicação dos profissionais de saúde, qualquer desatenção a esses pacientes graves pode ser fatal (POLKEY E e MOXHAM DF, 2013).

A rotina da UTI é intensa, sendo que a equipe de enfermagem deve estar desempenhada para qualquer instante, atender os pacientes com mudanças hemodinâmicas importantes, as quais carecem competências específicas e enorme prática para empunhar decisões e implementá-las em tempo rápido.

Pode-se presumir que a equipe exerça importante papel no âmbito da UTI. O cuidado intensivo dispensado a pacientes graves, torna-se mais eficiente quando desenvolvido em unidades específicas, que possibilita recursos e facilidades para a sua evoluída recuperação (GOMES AM, 2012).

O autor Sarmento VM (2014) aborda em seu estudo que a equipe de UTI precisa estar qualificada a executar atividades de enorme complexidade, para as quais é essencial a autoconfiança fundamentada e respaldada no entendimento científico para que este possa acompanhar o atendimento do paciente com segurança. A capacitação e o treinamento desse profissional são indispensáveis para o alcance do resultado esperado. 
A equipe de enfermagem tem o comprometimento de desempenhar suas atuações científicas e éticas, para alcançar este objetivo, faz-se fundamental ter qualificação, capacitação permanente e eficácia de conhecimento moral para a tomada de decisões dentro dos preceitos éticos do profissional. Esse tema é vasto e bastante debatido por inúmeros autores, todavia neste trabalho, pretende-se identificar e descrever a percepção da equipe de enfermagem a respeito do tema abordado (CARRARETTO AR, 2014).

Nas UTI's, a enfermagem envolve-se, diretamente, na assistência dos pacientes graves, é primordial ressaltar que a maior parte das complicações, procedentes do cuidado, poderiam ser evitadas, se a equipe desempenhasse a assistência adequada para lidar com o paciente crítico. A falta de percepção das ações da equipe associado ao paciente grave em UTI, pode tornar sua utilização uma razão de estres para toda a equipe da UTI (VIEIRA SRR, 2013).

O cuidado aos pacientes graves torna-se incumbência da equipe, pois a evolução positiva deles advém da assistência continuada, instruídos de proporcionar o reconhecimento de complicações que atinjam exatamente suas necessidades para uma contribuição de cuidado de qualidade é essencial que a equipe possua um vasto conhecimento dos fundamentos da assistência, além de distinguir a tolerância fisiológica específica de cada paciente (NÓBREGA MML, 2014).

A seriedade das ações equipe na UTI é indispensável para a eficácia da qualidade na assistência ao paciente e seus familiares, equipe encara cotidianamente as inúmeras necessidades associadas a dificuldade prática da assistência a ser executada, às imposições e exigências dos clientes, familiares, área médica, instituição, dentre outras (SARMENTO VM, 2014).

O estudo se propõe a responder a seguinte questão norteadora: Como a equipe de Enfermagem de uma Unidade de Terapia Intensiva Adulto descreve os cuidados ao paciente grave?"

A relevância desse estudo volta-se para a assistência à saúde ao paciente crítico em UTI, sendo que a equipe de enfermagem esteja apta a lidar com situações difíceis, em que terão de tomar decisões de maneira rápida e que garanta a segurança do paciente e de sua família, que muitas vezes precisa também de um suporte durante a internação. Com o objetivo tem como descrever a percepção da equipe de enfermagem quanto ao cuidado ao paciente grave em uma UTI adulta em um hospital privado de Porto Velho-RO.

\section{MÉTODOS}

Estudo descritivo-exploratório, bibliográfico e com abordagem qualitativa. A amostra compreendeu a totalidade da equipe de enfermagem que exercem suas funções no compreendendo: 20 profissionais de enfermagem. Os critérios de inclusão foram estabelecidos da seguinte forma quanto à população a ser pesquisada, foram todas as equipes de enfermagem da UTI adulto e que assinaram o Termo de Consentimento Livre e Esclarecido, no qual esteve na unidade no período compreendido da pesquisa.

Os critérios de exclusão foram os profissionais que pertencem à equipe de enfermagem em período de férias, licença ou afastamento durante o período da pesquisa, ausência em todas as tentativas de busca do profissional e os que não aceitarem em participar da pesquisa. O estudo foi desenvolvido obedecendo ao estabelecido na Resolução n. ${ }^{4}$ 466/2012/CNS/MS.

Em virtude de se tratar de uma pesquisa com seres humanos, o estudo foi submetido ao Comitê de Ética em Pesquisa (CEP) e após parecer favorável, iniciou-se a coleta de dados. A pesquisa foi realizada através da aplicação de um questionário contendo 08 (oito) perguntas abertas relacionadas a percepção da equipe de enfermagem quanto ao cuidado ao paciente grave em uma UTI.

O questionário foi entregue aos participantes e no mesmo momento do término do preenchimento foram recolhidos. Após a sua assinatura do termo foi então entregue o questionário. $O$ presente estudo foi submetido à apreciação do CEP da UNINORTE, o qual expediu parecer favorável sob o número 3.132.272, em 05 de fevereiro de 2019. 


\section{RESULTADOS E DISCUSSÃO}

Em relação a apresentação dos resultados e sua análise caracterizando os participantes desta pesquisa, a amostra foi composta por 4 enfermeiros e 16 técnicos em enfermagem, constituindo assim um total de 20 profissionais que aceitaram participar do estudo. As informações principais referentes à identificação dos profissionais de enfermagem que participaram da pesquisa estão dispostas na Tabela 1 abaixo, contendo a caracterização dos enfermeiros e dos técnicos em enfermagem. Observou-se que na Tabela 1 o respectivo setor existem 4 enfermeiros com predominância do sexo feminino, sendo três com idade entre 30 e 35 anos, e um do sexo masculino entre 24 e 29 anos. Dos 16 técnicos em enfermagem que participaram do estudo, dois estão entre 18 e 23 anos, dois entre 24 a 29 anos, sete entre 30 e 35 anos e cinco acima de 36 anos, quanto ao sexo, 15 são do sexo feminino, demonstrando mais uma vez a predominância do respectivo gênero.

Tabela 1 - Caracterização dos enfermeiros e técnicos em enfermagem que participaram da pesquisa. Porto Velho-RO, 2019.

\begin{tabular}{ccc}
\hline PROFISSIONAL & FAIXA ETARIA & GËNERO \\
\hline E1 & Entre 30 a 35 anos & $\mathrm{F}$ \\
E2 & Entre 24 a 29 anos & $\mathrm{F}$ \\
E3 & Entre 30 a 35 anos & $\mathrm{F}$ \\
E4 & Entre 30 a 35 anos & $\mathrm{M}$ \\
T1 & Acima de 36 anos & $\mathrm{F}$ \\
T2 & Acima de 36 anos & $\mathrm{F}$ \\
T3 & Entre 30 a 35 anos & $\mathrm{F}$ \\
T4 & Entre 18 a 23 anos & $\mathrm{F}$ \\
T5 & Entre 24 a 29 anos & $\mathrm{F}$ \\
T6 & Entre 30 a 35 anos & $\mathrm{M}$ \\
T7 & Entre 30 a 35 anos & $\mathrm{M}$ \\
T8 & Acima de 36 anos & $\mathrm{F}$ \\
T9 & Entre 30 a 35 anos & $\mathrm{F}$ \\
T10 & Entre 30 a 35 anos & $\mathrm{M}$ \\
T11 & Entre 30 a 35 anos & $\mathrm{M}$ \\
T12 & Entre 30 a 35 anos & $\mathrm{F}$ \\
T13 & Acima de 36 anos & $\mathrm{F}$ \\
T14 & Acima de 36 anos & $\mathrm{F}$ \\
T15 & Entre 18 a 23 anos & $\mathrm{F}$ \\
T16 & Entre 24 a 29 anos & $\mathrm{F}$ \\
\hline
\end{tabular}

Fonte: Bezerra JM e Fonseca IAC, 2019.

Françolin MT et al. (2015) destacaram em seu estudo realizado em seis hospitais de Ribeirão Preto, São Paulo que dos 49 profissionais de enfermagem pesquisados, 47 eram do sexo feminino.

Souza et al. (2015) confirmam em sua pesquisa que três unidades de ensino do sul do Brasil, 47 dos 71 profissionais de enfermagem também eram do sexo feminino.

Almeida (2012) afirma que a enfermagem, por ser uma profissão de predomínio feminino, confere à mulher a oportunidade de gerenciar, não sendo comuns em nossos meios profissionais questionamentos sobre a capacidade administrativa das mulheres nesta área.

\section{Achados relevantes e categorias temáticas levantadas}

A análise de conteúdo das respostas foi identificada como parte fundamental do nosso processo de investigação como estratégia de compreensão, a partir da percepção dos profissionais de enfermagem, como eles veem o cuidado ao paciente grave na UTI adulta.

Das respostas analisadas emergiram quatro grupos temáticos e ideias-chaves. No primeiro grupo levantado, a Avaliação da equipe de enfermagem ao cuidado prestado ao paciente grave, será 
discutida: a percepção dos profissionais de enfermagem quanto à ao cuidado ao paciente grave na UTI. O segundo grupo abordará sobre as Facilidades no cuidado ao paciente grave, onde será feita uma avaliação pelos profissionais em relação as facilidades na assistência ao paciente grave. O terceiro grupo, as Dificuldades no cuidado ao paciente grave, que discutirá sobre os desafios dos profissionais nos cuidados prestados a esses pacientes graves na UTI adulta (Quadro 1).

Ao final abordaremos o grupo temático levantando os desafios da equipe de enfermagem sobre os cuidados prestados ao paciente grave em UTI, e nele serão trabalhadas as principais relevâncias encontradas pelos profissionais de enfermagem e os desafios para a equipe de enfermagem nos cuidados prestados a esses pacientes (Quadro 1).

Quadro 1 - Categorias temáticas e ideias-chaves levantadas, referente a percepção da equipe de enfermagem sobre o cuidado ao paciente grave. Porto Velho, Rondônia, 2019.

\begin{tabular}{|l|l|}
\hline \multicolumn{1}{|c|}{ Categorias temáticas } & \multicolumn{1}{c|}{ Ideias-chaves } \\
\hline $\begin{array}{l}\text { Avaliação da equipe de enfermagem ao } \\
\text { cuidado prestado ao paciente grave }\end{array}$ & $\begin{array}{l}\text { A percepção dos profissionais de enfermagem quanto à ao } \\
\text { cuidado ao paciente grave na UTI }\end{array}$ \\
\hline $\begin{array}{l}\text { Facilidades no cuidado ao paciente } \\
\text { grave }\end{array}$ & $\begin{array}{l}\text { Avaliação pelos profissionais em relação as facilidades na } \\
\text { assistência ao paciente grave }\end{array}$ \\
\hline $\begin{array}{l}\text { Dificuldades no cuidado ao paciente } \\
\text { grave }\end{array}$ & $\begin{array}{l}\text { Desafios dos profissionais nos cuidados prestados a esses } \\
\text { pacientes graves na UTI adulta. }\end{array}$ \\
\hline $\begin{array}{l}\text { Os desafios da equipe de enfermagem } \\
\text { sobre os cuidados prestados ao } \\
\text { paciente grave em UTI }\end{array}$ & $\begin{array}{l}\text { As principais relevâncias encontradas pelos profissionais de } \\
\text { enfermagem e os desafios para a equipe de enfermagem nos } \\
\text { cuidados prestados a esses pacientes. }\end{array}$ \\
\hline
\end{tabular}

Fonte: Bezerra JM e Fonseca IAC, 2019.

\section{Avaliação da equipe de enfermagem ao cuidado prestado ao paciente grave na UTI}

Neste grupo temático, descreveremos a percepção dos profissionais em relação a avaliação da equipe de enfermagem ao cuidado prestado ao paciente grave na UTI.

Chaves AS et al. (2012) afirmam que a equipe de enfermagem desempenha papéis fundamentais dentro da unidade de terapia intensiva, através da obtenção do histórico do paciente, realização de exame físico, supervisão da equipe de enfermagem, avaliação das condições clínicas do cliente, orientações e planejamento diário das ações de enfermagem, procurando sempre otimizar a assistência humanizada com o aparato tecnológico.

Bergamini MEO (2013), evidencia em seu estudo que o ambiente da UTI exige atitudes individuais e coletivas dos profissionais no sentido de avaliar o cuidado prestado, respeitando a individualidade, a dignidade e a privacidade do cliente, levando em consideração os princípios éticos, ou seja, a equipe deve adotar medidas conscientes e ordenadas, que minimizem o desconforto e o sofrimento do paciente, considerando assim, os seus direitos.

Caetano JA et al (2013) afirma em seu estudo que mesmo que a equipe de enfermagem ofereça uma assistência de qualidade nas técnicas e procedimentos, pode ser insuficiente em relação ao cuidado prestado, com isso a literatura cientifica aborda a escassez de tempo, desmotivação e acumulação de atividades. Para cuidar do paciente, antes, é preciso cuidar de si próprio, pois a assistência só acontece com a transferência de sentimentos com a intuição de ajuda o paciente.

Ao abordar o tema avaliação da equipe de enfermagem ao paciente grave em UTI, pode-se observar nas falas dos participantes a seguir:

"Buscar a excelência no atendimento para melhorar a qualidade de vida do paciente internado". (T2)

"Boa qualidade, pois a equipe de enfermagem presta uma assistência de qualidade ao paciente". (E2) 
Os profissionais durante a abordagem e coleta de dados, tornou-se a pesquisa mais produtiva e foi possível elencar pontos relevantes sobre o cuidado prestado ao paciente grave em UTI, bem como a importância que o profissional tem na assistência, quando as técnicas são executadas conforme o que é preconizado, visando assim à segurança do paciente (OLIVEIRA PL, 2016).

Frotes AR (2015) enfatiza que o cuidado prestado é calçado nos princípios da ética. Isso significa valorizar os sujeitos que participam do processo de cuidar, favorecendo o desenvolvimento de sua sensibilidade e competência, reconhecendo a singularidade de cada um e seus potenciais na busca de estratégias que viabilizem um cuidado que leve à recuperação do cliente em todas as suas dimensões.

Abordar o cuidado prestado no paciente grave em UTI não se restringe somente a técnica, no fazer, mas também no sentido de ser, de expressar uma atitude, pois existe uma relação entre a equipe de enfermagem, paciente e família (GONÇALVES S, 2016).

Nota-se que para que o atendimento ocorra com qualidade e de forma segura, deve-se ter a provisão de materiais, equipamentos, recursos humanos especializados e a notificação de Eventos Adversos dentro da unidade (VIEIRA SRR, 2013).

A avaliação do paciente está ligada diretamente à qualidade na prestação do cuidado, pois um serviço só tem qualidade se os riscos de danos ao paciente estiverem diminuídos, por isso se faz necessário ter uma ambiência correta e materiais suficientes (BRASIL, 2013).

Carvalho DF e Cassiani SA (2012) acreditam que para melhorar a avaliação do paciente, é imprescindível implementar uma política institucional. Inicialmente, para que essa mudança ocorra, é essencial o levantamento dos fatores organizacionais que a impedem, para posteriormente investir em capacitações no ambiente.

Evidencia-se nos resultados da pesquisa, a fala do E4 que reforça a importância de uma avaliação da equipe ao cuidado prestado ao paciente grave:

"Avaliação dos cuidados prestados em relação ao paciente grave na UTI são bons" (E4).

Salienta se ainda que um ambiente adequado e com recursos necessários, auxiliam no processo de trabalho, pois ocorre uma mudança no comportamento dos funcionários que passarão a atuar de forma mais segura e correta, uma vez que tem uma estrutura que favorece o atendimento da forma que é preconizada (FROTES AR, 2015).

\section{Facilidades no cuidado ao paciente grave na UTI}

No que tange as facilidades ao cuidado ao paciente grave na UTI Adulto, de acordo com a equipe de enfermagem, foi apontado principalmente o fato de todos os pacientes estarem monitorizados, por meio de monitores eletrônicos, nos leitos. Isso se mostrou primordial e de extrema importância não só para a qualidade de atenção ao paciente como também para o trabalho da equipe em geral, como se observa nas falas dos participantes a seguir:

"Ter paciente monitorizado, acho que é fácil trabalhar". (E1)

"Os pacientes estão monitorizados, isso facilita o trabalho". (E2)

Fernandes SD (2016) destaca que a assistência aos pacientes na UTI está relacionada ao cuidado direto, intensivo e ao monitoramento permanente por parte dos profissionais. No entanto, o cuidado na UTI requer não só um cuidado técnico, voltado apenas para a dimensão biológica, mas um cuidado integral com os pacientes, tratando-os como seres humanos, com respeito, afetividade e dedicação, conversando com os mesmos, consolando-os quando necessário e vendo sempre o que é melhor para eles, fazendo com que os pacientes se sintam bem cuidados em todos os sentidos, mesmo estando em coma, sedados ou inconscientes. 
Oliveira PL (2016) ressalta que para a equipe de enfermagem, o uso de monitores multiparâmetros para o controle de sinais vitais dos pacientes, facilita o trabalho de toda equipe, uma vez que apresentam cores e sons específicos para alertar em caso de alguma emergência, além de permitirem rápida e fácil visualização dos parâmetros vitais.

Na pesquisa de Fonseca AS (2015), a tecnologia vem sendo muito utilizada em terapia intensiva, fornecendo benefícios tanto para o paciente quanto para os que realizam o cuidado. A tecnologia auxilia legitimando as condutas profissionais, que podem ser utilizadas como critério de avaliação da qualidade dos serviços de saúde prestados pelos hospitais.

As UTIs são as unidades mais complexas e mecanizadas de um hospital por apresentar todo o arsenal tecnológico necessário para assistir pacientes graves, e o manuseio desses equipamentos avançados exige recursos humanos com competência para realizar tais atividades.

Estudos consideram a tecnologia dura como fator importante para o cuidado com qualidade, mas não é o principal. É importante considerar a tecnologia leve, as relações e o vínculo para embasar o cuidado efetivo com qualidade (BASTOS IAP, 2015).

A presença de médicos e da equipe de enfermagem 24 horas por dia na unidade gera segurança e conforto para a equipe facilitando uma assistência adequada. A disponibilidade desses trabalhadores mostra-se fundamental em um ambiente de pacientes hemodinamicamente instáveis (OLIVEIRA PL, 2016).

As seguintes falas retratam essa situação:

"O Médico está presente 24 horas, isso é fundamental e uma equipe sempre disponivel”. (E2)

"A enfermagem à disposição, então isso facilita muito, tudo próximo, o acesso é bem mais fácil". (T6)

Nos relatos acima foi possível evidenciar o quanto essas características peculiares de unidades menores, onde se realizam cuidados intensivos, mostram-se de extrema importância para quem cuida, pois o fato de haver médico e a equipe de enfermagem durante as 24 horas do dia traz conforto e sensação de apoio para esses sujeitos.

O acesso para sanar possíveis dúvidas torna-se mais fácil e rápido, o que contribui de forma positiva tanto para o profissional, ao prestar assistência, quanto para o paciente (SILVA DC, 2016).

Aliado a isso, a disponibilidade de materiais oferece melhor qualidade de cuidado e segurança para o trabalhador, ao desempenhar suas tarefas.

Um dos fatores citados, pelos entrevistados, que contribui, positivamente, para o desenvolvimento do trabalho é 0 acesso fácil e rápido à aparelhagem, aos materiais e às medicações necessárias para 0 atendimento de pacientes instáveis (SILVA CDMB e CARVALHO MAM, 2014).

$\mathrm{Na}$ UTI o objetivo comum é a recuperação do paciente em tempo hábil, num ambiente físico e psicológico adequados, onde a atitude particular de cada membro da equipe que ali trabalha está orientada para o aproveitamento das facilidades técnicas existentes, aliadas a um bom relacionamento humano (KELIN LA, 2016).

Durante a coleta, foi possível identificar diversos tipos de facilidades no cuidado ao paciente grave em UTI na visão da equipe, o que foi confirmado pelas falas a seguir:

"Desenvolver o trabalho com competência e habilidade técnica e humana que corresponda às necessidades dos pacientes". (T16)

"Treinamentos, educação continuada, número suficiente de profissionais". (T11)

Estes fatores positivos é que facilitam os cuidados ao paciente grave em UTI, nota-se que muitos destes aspectos considerados positivos, e que são provenientes da necessidade de adaptação das instituições de 
saúde e estão relacionados a mudanças e (re) organizações da estrutura física e das rotinas dos serviços (SANTOS AK, 2014). Kelin LA (2016) enfatiza que o trabalho em equipe se apresenta como um fator de extrema importância, para que se possa oferecer um cuidado de qualidade. Os trabalhadores apontam que, para isso, faz- -se necessário que cada um da equipe tenha consciência da responsabilidade de seu trabalho. A cooperação e a harmonia entre a equipe de enfermagem surgem, como aspectos positivos, como se observa a seguir:

"A atividade em equipe facilita muito. $A$ evolução em si porque é um trabalho que tem uma continuidade para poder dar assistência para o paciente". (E3)

"A equipe é um fator que facilita bastante, ter uma equipe dinâmica é excelente". (E1)

O êxito desse trabalho coletivo depende do desempenho de cada um, e seu resultado só será satisfatório mediante o desenvolvimento de um bom trabalho individual. Sob esse aspecto, aponta-se que, cada profissional, precisa assumir a sua parcela de comprometimento no sentido de manter o ambiente de trabalho organizado (SILVA DC, 2016).

Silveira MAM et al. (2015) retratam que quando isso não ocorre, acontece a sobrecarga de trabalho para alguns trabalhadores que, apesar de realizá-lo, o fazem com sofrimento. Trabalhar em equipe é importante, pois propicia a ajuda mútua e o companheirismo, seja no momento de cuidar dos pacientes, ou na hora de cuidar de um colega. Ao se trabalhar em equipe desenvolve-se a comunicação contínua, estabelece-se a cooperação, propicia-se a democracia e a concentração nos objetivos comuns.

\section{Dificuldades no cuidado ao paciente grave}

No que tange as dificuldades no cuidado ao paciente grave em UTI adulto, os participantes destacam os conflitos pessoais, decorrentes do convívio em ambiente fechado, uma vez que, a equipe de enfermagem passa grande parte do turno de trabalho, confinados num local pequeno, convivendo com os mesmos colegas quase que diariamente (FONSECA AS, 2015).

Dividem momentos alegres e tristes e afirmam que, para se obter uma boa relação interpessoal entre os membros da equipe de enfermagem, é fundamental o bom convívio para o desempenho de seu trabalho, como relatado por E2 e ratificado por E3:

"Por ser uma unidade fechada, vive sempre convivendo com os mesmos profissionais, pode acontecer um problema com a equipe e por ser uma coisa mais fechada, mais confinado naquele ambiente, a equipe divide as alegrias $e$ as tristezas junto com as pessoas". (E2)

"Eu vejo UTI, uma unidade fechada, a interação interpessoal é direta, se não tem um bom relacionamento as coisas complicam, o trabalho não anda porque não tem como por ser um o espaço físico é muito pequeno". (E3)

Os depoimentos acima sugerem que as relações interpessoais influenciam, fortemente, no decorrer do trabalho. Desse modo, os conflitos entre os membros da equipe podem gerar falta de comprometimento com o trabalho ou até mesmo frustração de alguns trabalhadores.

Sabe-se que o processo de trabalho em UTI e suas características influenciam, diretamente, no relacionamento da equipe. Para a construção do trabalho em equipe, é necessário o enfrentamento dialógico de conflitos, buscando a flexibilidade das regras, negociações e acordos entre os agentes, e requer compartilhar decisões e responsabilidades (SANTOS AK, 2014).

O trabalho em equipe pode ser facilitado através de atitudes de respeito e cordialidade entre todos os membros, por meio de atitudes de aceitação e tolerância de diferentes visões com postura ética, verificou-se que, alguns participantes não admitiram este problema, enquanto outros mencionam que estão começando ou tentando se relacionar melhor com os colegas de profissão (FONSECA AS, 2015). 
Aliada a essas dificuldades de relacionamento entre os colegas de trabalho, surge também, como um obstáculo enfrentado pelos participantes, o envolvimento emocional com os pacientes, e concomitante a isso, a fuga e o afastamento dessa situação, como se observa nos depoimentos:

"No início se abala muito, mas depois aprende a separar". (T9)

"Acho que o emocional mesmo, eu acho que isso é um fator negativo". (T7)

Silva DC (2016) descreve em seu estudo que o ser humano é atribuído de sentimentos e emoções, o tornando-o emotivo e frágil ao sofrimento do paciente. A equipe de enfermagem sente-se ainda mais estimulada porque tem a função de cuidar do paciente que está em estado crítico.

\section{Os desafios da equipe de enfermagem sobre os cuidados prestados ao paciente em UTI}

Descreveremos a seguir, as principais relevâncias encontradas pelos profissionais de enfermagem e os desafios para a equipe de enfermagem nos cuidados prestados a esses pacientes.

A pesquisa de Freitas PG (2016) comprova que apesar de ter inúmeros profissionais que exerce na UTI a equipe é o responsável pela assistência continuada, consequentemente tem o comprometimento de estabilizar a homeostasia do cliente e o bom andamento do setor.

Mendes AS et al. (2017) acreditam o cuidado de enfermagem intensivo se constitui em um contínuo processo de aperfeiçoamento pelo fato dos clientes adultos em estado crítico apresentarem curso clínico altamente instável, com elevado risco de morte e em condições de saúde sujeitas às frequentes variações.

Assim, profissionais cada vez mais capacitados, que possam prontamente identificar essas necessidades humanas básicas afetadas e utilizar o processo de enfermagem na UTI para aprimorar a sua tomada de decisões, se faz necessário para especificar cuidados adequados a cada cliente, formular planejamentos específicos, fazer prescrições de enfermagem eficazes e avaliar o estado de saúde por meio de evoluções (SILVA DC, 2016).

É possível destacar que dentro do da UTI existem diversos cuidados que devem ser vencidos no dia a dia, um deles é no que diz respeito à estrutura, déficit de recursos humanos e insumos (CAETANO JA et al., 2013). Tais problemas tornam a assistência mais difícil de ser executada de uma maneira satisfatória, os quais podem analisar a partir das falas a seguir:

"Um ambiente limpo, agradável, falta de materiais, leitos, medicamentos e recursos humanos é o grande desafio para uma assistência adequada". (T6)

Todos os problemas elencados pelos profissionais de enfermagem do setor são relevantes, pois contribuem para que os eventos adversos ocorram, uma vez que a assistência não pode ser prestada da maneira correta por falta de recursos materiais, físicos e humanos, sendo que tais problemas dificultam o processo de trabalho (FONSECA AS, 2015).

Silva DC et al. (2016) corroboram com as falas supracitadas dizendo que as instituições de saúde brasileiras vêm enfrentando falta de planejamento em saúde, problemas de rotatividade de profissionais, recursos humanos escassos e deficitários e falhas na estrutura física.

Berti GRS (2015) afirma que desafios relacionados às falhas na estrutura física e à falta ou quantidade insuficiente de equipamentos e materiais para atender às necessidades também aparecem como adversidade no ambiente de trabalho.

No estudo evidencia-se que o ambiente e o atendimento influenciam nas práticas de enfermagem, por isso faz-se necessário melhorar a ambiência e condições de trabalho para que haja uma assistência segura e de forma digna (FROTES AR, 2015).

A equipe de enfermagem da UTI deve dispor de entendimento, prática e conduta, incumbe ao enfermeiro sistematizar e determinar sobre a utilização de recursos humanos, físicos, materiais e referência nos cuidados desempenhados (BERTI GRS, 2015). 
No local de uma UTI, estas atribuições estão associadas a assistência com o paciente em estado grave que engloba um sistema tecnológico exclusivo, requerendo dos enfermeiros percepção e competências associadas ao manuseamento de máquinas e às necessidades dos pacientes graves que precisam delas (SCHWONKE DF et al., 2015).

O diálogo efetivo com o paciente dentro da UTI é um dos obstáculos da equipe, principalmente quando o cliente se encontra em traqueostomia, o empenho e senso são ainda os melhores instrumentos (CAETANO JA et al., 2013).

A equipe de Enfermagem deve ter suporte de entendimentos que simplifique a capacidade de compreender uma enorme diversidade de questões, bem como referências elevadamente estabelecidas e especificas, pois o enfermeiro deve ser o mais auto realizado possível: físico, emocional e espiritual a fim de atender os desafios de oferecer assistência aos pacientes graves (FONTES NM et al., 2014).

Outros obstáculos e desafio da equipe de enfermagem é estar capacidade e qualificada para manuseios e funcionamento de equipamentos e aptos teoricamente sob a ótica cognitiva e de habilidades para prestar uma assistência ao paciente grave (BERTI GRS, 2015).

De acordo com Freire AS (2016) em sua pesquisa evidencia que o propósito da assistência prestada é sempre proporcionar um igualo fisiológico e emocional ao cliente, a equipe precisa entender e analisar cada vez mais a condição humana e promovendo o respeito à autonomia e dignidade de cada um.

\section{CONCLUSÃO}

Os cuidados prestados pela equipe de enfermagem aos pacientes graves em Unidades de Terapia Intensiva são de suma importância, além disso, para uma boa prática assistencial é preciso haver um cuidado mais holístico visando não apenas os cuidados físicos e biológicos, mas também, aqueles de caráter psicológico e social, promovendo, dessa forma, uma assistência adequada. Diante de todas estas evidências, conclui-se que para tornar o cuidado humanizado, não basta investir somente na eficiência técnico-científica. É preciso, também, atentar-se a princípios e valores como a solidariedade e a ética na relação entre a equipe, a família e o próprio paciente, acolhendo-os, respeitando e aceitando os limites de cada situação, sempre respeitando o próximo.

\section{REFERÊNCIAS}

1. ALMEIDA PG. A importância do enfermeiro na promoção da humanização na unidade de terapia intensiva. Revista Eletrônica de Enfermagem do Centro de Estudos de Enfermagem e Nutrição. v.1, n.1 Jan-jul. São Paulo. 2012.

2. BASTOS IAP. Cuidado humanizado de enfermagem: o agir com respeito em um hospital universitário. Revista Brasileira de Enfermagem. v.1, n.2. São Paulo. 2015.

3. BERGAMINI MEO. Estressores vivenciados por pacientes de terapia intensiva e suas estratégias de enfrentamento: um estudo direcionado à assistência de enfermagem. [tese] Rio de Janeiro: Universidade Federal do Rio de Janeiro, Escola de Enfermagem Ana Nery; 2013.

4. BERTI GRS. A unidade de terapia intensiva: um estudo sobre a comunicação entre profissionais e pacientes. [tese]. São Paulo: Escola Paulista de Medicina - Universidade Federal de São Paulo; 2015.

5. BRASIL. Caracterização da população atendida em Unidade de Terapia Intensiva: subsídio para a assistência. Brasília. 2013.

6. CARVALHO DF, CASSIANI SA. Experiência de pacientes internados em UTI. [tese]. Ribeirão Preto: Escola de Enfermagem de Ribeirão Preto, Universidade de São Paulo; 2012.

7. CAETANO JA, et al. Cuidado humanizado em terapia intensiva: um estudo reflexivo. Escola Anna Nery Revista de Enfermagem. São Paulo. 2013.

8. CARRARETTO AR. Paciente crítico em UTI. Curso de Educação à Distância em Anestesiologia. São Paulo: 2014. 
9. CHAVES AS, PORTO VC, VERISSÍMO CV. O estresse da equipe de enfermagem na unidade de terapia intensiva: o enfermeiro como mediador. Arquivos Catarinenses de Medicina v. 35, n. 4, p. 36-43, Florianópolis. 2012.

10. FERNANDES SD. Discursos de enfermeiros sobre humanização na unidade de terapia intensiva. Esc Anna Nery, v. 16, n. 4, p. 719-727, São Paulo. 2016.

11. FONSECA AS. O clínico e as manifestações psíquicas no paciente de UTI. In: Anais do 1(Congresso Brasileiro De Psiquiatria e Medicina Interna. São Paulo: Editora Astúrias; 2015. 333p.

12. FONTES NM. et al. Desafios e estratégias das enfermeiras na Unidade de Terapia Intensiva neonatal. Rev. Rene. Fortaleza, v. 11, n. 4, p. 76-84, out./dez., 2014.

13. FRANÇOLIN MT. O cuidado intensivo oferecido ao paciente no ambiente de Unidade de Terapia Intensiva. Rev Esc Anna Nery, v. 16, n. 4, p. 689-96, São Paulo. 2015.

14. FREITAS PG. A importância do enfermeiro na promoção da humanização na unidade de terapia intensiva. Revista Eletrônica de Enfermagem do Centro de Estudos de Enfermagem e Nutrição. v.1, n. 2. Jan-jul. São Paulo. 2016.

15. FREIRE SA. Assistência prestada pelo enfermeiro em unidades de terapia intensiva: aspectos afetivos e relacionais. [dissertação]. Ribeirão Preto (SP): Escola de Enfermagem/USP; 2016.

16. FROTES AR. Pacientes de UTI: perspectivas e sentimentos revelados. Nursing, v. 6, n. 57, p. 12-17, São Paulo. 2015.

17. GOMES AM. Enfermagem na unidade de terapia intensiva, 2 ed., São Paulo, EDU, 2012. 140p.

18. GONÇALVES S. Percepções de profissionais de saúde sobre a humanização em unidade de terapia intensiva adulto. Esc Anna Nery, v. 1, n. 2, São Paulo. 2016.

19. KELIN LA. O significado cultural do cuidado humanizado em unidade de terapia intensiva: "muito falado e pouco vivido". Rev Latino-Am Enf, v.1, n.2. Ribeirão Preto, 2016.

20. LINO MM, SILVA SC. Enfermagem na Unidade de Terapia Intensiva: a história como explicação de uma prática. Nursing, 2013.

21. OLIVEIRA PL. A percepção do paciente crítico sobre sua permanência na unidade de terapia intensiva. Rev Esc Enfermagem da USP, v.1, n.2. São Paulo, 2016.

22. MENDES AS et al. UTI: Muito além da técnica... a humanização e a arte do intensivismo. 5 ed. Atheneu, 2017. 250p.

23. NASCIMENTO ERP, TRENTINI M. O cuidado de enfermagem na unidade de terapia intensiva (UTI): teoria humanística de Paterson e Zderad. Rev Latino-am Enfermagem. São Paulo. 2012.

24. NÓBREGA MML de. Conhecimento na Enfermagem. Revista Nursing-Edição Brasileira: Editorial Bolina Brasil, $\mathrm{n}$. 110, p.293I, jul. 2014.

25. POLKEY E, MOXHAM DF. Enfermagem em terapia intensiva. São Paulo: Atheneu. 2013. 158p.

26. RICA CV, TURATTO VB. Reações emocionais de pacientes internados em UTI por tempo prolongado. In: Anais do 1(Congresso Brasileiro de Psiquiatria e Medicina Interna. São Paulo: Editora Astúrias; 2016. p. 124-5

27. SARMENTO VM. Enfermagem em Unidade de Terapia Invasiva, retrospectiva histórica. São Paulo. 2014.

28. SANTOS AK. O paciente em centro de terapia intensiva: reflexão bioética. Rev. Esc. Enf. USP, v.1, n.1. São Paulo, 2014.

29. SILVA DC. A enfermagem e as manifestações psíquicas nos pacientes de UTI. In: Anais do 1(Congresso Brasileiro de Psiquiatria e Medicina Interna. São Paulo: Editora Astúrias; 2016.

30. SILVA CDMB, CARVALHO MAM. O significado de humanização da assistência para enfermeiros que atuam em UTI. Rev. Eletr. Enf. São Paulo, 2014.

31. SILVEIRA MAM. et al. O significado de humanização da assistência para enfermeiros que atuam em UTI. Cuiabá-MT. 2015.

32. SCHWONKE DF et al. Assistência de Enfermagem ao paciente gravemente enfermo. EPU, 2 ed. São Paulo, 2015. 235p.

33. VIEIRA SRR. UTI na Enfermagem - pacientes graves: Programa de Atualização de Medicina Intensiva Brasileira. Porto Alegre: Artmed/Panamericana, 2013. 